\title{
Logistics management based on Optimization Algorithm
}

\author{
Liu Lijuan \\ Yunnan Vocational Institute of Energy Technolgy, Yunnan Qujing, China, 655000
}

Keywords: logistics management; optimization algorithm; analysis

Abstract: SMEs play an increasingly important role in promoting national economic development and social progress. With increasingly fierce market competition, the challenges faced by SMEs are gradually increasing, and it is urgently needed to improve logistics management to increase the economic benefits of enterprises. Although SMEs are not strong in strength, the impact of logistics activities on companies continues to increase, and companies must also pay attention to logistics construction. This paper elaborates the problems existing in SMEs in light of the major problems in the logistics management of SMEs, and analyzes the optimization algorithms of logistics management to lay a solid foundation for further development.

\section{Introduction}

The analysis of the concept of logistics from the field of economics can be simply summarized by providing ways to solve the problem of time and space circulation differences in products, and to promote the smooth conduct of economic activities through the elimination of time and space barriers in economic activities. However, from the perspective of management, logistics is the planning and control of the product supply chain of an enterprise. Therefore, the logistics activity is not only the transportation of products, but also the management of the economic activities of the enterprise by controlling the product supply chain[1].

\section{Major issues in the logistics management of SMEs}

\subsection{Concept Behind}

The scale and strength of small and medium-sized enterprises determine that in the process of development, the enterprise must focus on economic efficiency. It attaches great importance to the production and sales of related businesses, but it is relatively loose for auxiliary services such as logistics management. Increasing economic efficiency and accumulating capital are important for SMEs, but logistics management is also very important for SMEs. Even the degree of importance becomes more and more important with the construction of modern information-based businesses. So far, there are many small and medium-sized enterprises to understand the concept of logistics management in product transportation, simply thought that as long as the production of the production of materials shipped to the workshop before the products shipped out without delaying the market sales on it. This one-sided emphasis on production management, ignoring the importance of logistics management, will eventually increase logistics costs without doubt, has 
many adverse effects on the later development of SMEs. Can not be ignored is that logistics costs are also part of the cost of enterprises, logistics costs are high, will inevitably lead to the overall level of corporate profits[2].

\subsection{Logistics Management Needs to Improve in Means and Methods}

It is undeniable that many small and medium-sized enterprises are lagging behind in the way of logistics management. They still adopt traditional logistics methods and management methods. They place too much emphasis on the timely transportation of production raw materials and production products, and on what kind of transport methods they use for transport. The proportion of expenses in terms of profit has not been attached great importance; even some SMEs simply equate logistics with the transportation of production materials and products, exclude data analysis and enterprise inventory status in logistics management, and manage enterprise inventory. The status and role of logistics management cannot be measured objectively. The existence of these problems in the management methods and methods of SMEs logistics management undoubtedly increases the cost of raw materials and storage of products, and reduces the company's profit margins.

\section{Optimization Strategy for SME Logistics Management}

\subsection{Establish and improve the relevant logistics management system and constantly innovate management concepts}

Influenced by many factors such as the economic strength and development scale of SMEs, many managers and managers of enterprises only focus more on how to develop markets and improve economic efficiency. They are not sufficiently aware of the importance of logistics management. There is still a certain bias in the concept. For SMEs, we must change the traditional ideas and concepts, gradually increase the awareness of logistics management in the survival and development of enterprises, and establish a logistics management system suitable for enterprise development. Innovating the concept of logistics management requires SMEs to firmly establish modern logistics management concepts, fully recognize the importance of logistics in the growth of corporate profits, and to be good at thinking about innovative logistics management, not simply equating logistics with corporate cargo transportation. From the comprehensive consideration of many aspects such as production, operation, sales, etc., the advanced logistics management concept is carried through to the company's transportation, storage, procurement, processing, sales and other aspects, and the company's different production and operation links are scientifically arranged; at the same time, the company must formulate A practical and feasible logistics management system strictly controls the logistics costs in accordance with the system requirements and strengthens the company's cost accounting to better reduce logistics costs and expand the company's profit margins[3].

\subsection{Establishing a Small and Medium-sized Enterprise Logistics Information System}

The production, transportation, warehousing, sales, and logistics management of SMEs have involved all aspects. The diversification and complexity of control have limited the level of SMEs' logistics development. The use of human capabilities to achieve the development of SMEs' logistics management has become increasingly modern. Go farther. The modern logistics management of small and medium-sized enterprises must establish a network information database and use the network office system to manage the logistics information of small and medium-sized enterprises. Establishing a logistics information system for small and medium-sized enterprises is an important direction for the company's future development. SMEs start with warehouse management, establish 
an information warehouse management platform, and strengthen information management capabilities through data entry and retrieval. The warehouse system is a simple information template. The management personnel and staff can use the simple operation module to let the SME owner understand the logistics management information. Small and medium-sized enterprises can use the information system for the first time to check out the storage system, the reporting system, and the inventory system. On this basis, they can then contact the procurement plan, production plan, and financial system. Logistics management information system as shown below.

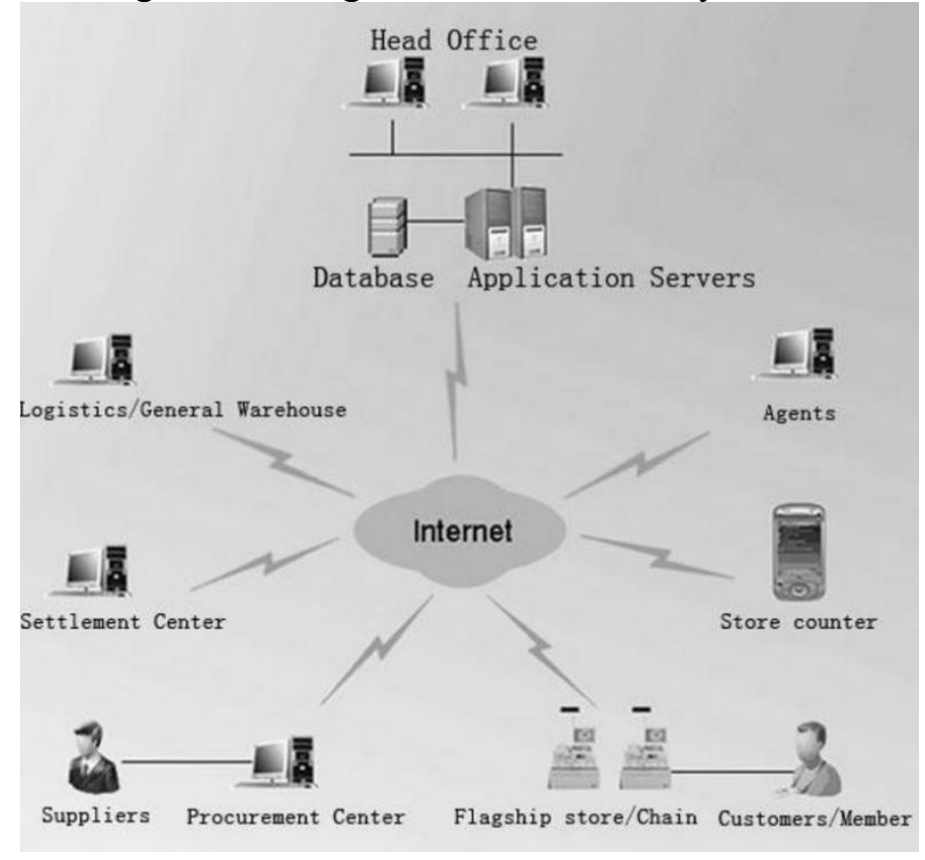

Fig.1 Logistics management information system

Using advanced algorithms, tabu search technology is a sub-heuristic search technique. The so-called taboo is to prohibit the repetition of previous work. In order to avoid the main inadequacies of local neighborhood search into local optimum, the tabu search algorithm uses a tabu list to record After reaching the local optimum, in the next search, these points are no longer searched using the information in the taboo list to jump out of the local optimum. It is like a person's short-term memory. The path taken is not repeated or selectively repeated. At the same time, "forgetting" makes these prohibitions a weak prohibition, that is, after a certain period of time, these prohibitions will be invalidated, and ultimately achieve the purpose of global optimization[4]. The TS algorithm has achieved great success in the areas of combinatorial optimization, production scheduling, machine learning, circuit design, and neural networks. It also gains a lot of research in the global optimization of functions, and has great development. The main calculation flow of the tabu search method: it can be divided into the starting solution, the definition of the neighborhood, the design of the taboo list, and the evaluation after the move. An initial solution is used as a starting point for the search, followed by a search procedure. In the search process, in order to avoid the occurrence of a cyclical phenomenon, a tabu list is created to record the search process. The structural characteristics and length of the taboo list can avoid the phenomenon of regional solution during the solution process. However, the taboo list may limit the scope of the solution, so it must be overcome by using forbidden criteria. The tabu search algorithm is the core of the tabu search algorithm design, including the tabu search object, the tabu length, the neighborhood structure, the evaluation function and the candidate set, and the ad hoc criterion. It also includes the determination of the amnesty rule and the termination rule. Disadvantages: The tabu search algorithm has a strong dependence on the initial solution. If the initial solution is poor, the convergence speed of the tabu 
search algorithm will decrease, and because the iterative search process is serial, it is only a single state of movement, not a parallel search. This affects the optimization efficiency. The logistics management optimization algorithm formula is as follows.

$$
B=K^{-T} K^{-1}=\left[\begin{array}{llll}
B_{11} & B_{12} & B_{13} \\
B_{21} & B_{22} & B_{23} \\
B_{31} & B_{32} & B_{33}
\end{array}\right]=\left[\begin{array}{ccc}
\frac{1}{\alpha^{2}} & -\frac{\gamma}{\alpha^{2} \beta} & \frac{v_{0} \gamma-u_{0} \beta}{\alpha^{2} \beta} \\
-\frac{\gamma}{\alpha^{2} \beta} & \frac{\gamma^{2}}{\alpha^{2} \beta}+\frac{1}{\beta^{2}} & -\frac{\gamma\left(v_{0} \gamma-u_{0} \beta\right)}{\alpha^{2} \beta^{2}}-\frac{v_{0}}{\beta^{2}} \\
\frac{v_{0} \gamma-u_{0} \beta}{\alpha^{2} \beta} & -\frac{\gamma\left(v_{0} \gamma-u_{0} \beta\right)}{\alpha^{2} \beta^{2}}-\frac{v_{0}}{\beta^{2}} & \frac{\left(v_{0} \gamma-u_{0} \beta\right)^{2}}{\alpha^{2} \beta^{2}}+\frac{v_{0}}{\beta^{2}}+1
\end{array}\right]
$$

\subsection{Ways to Improve Logistics Management}

The best way for SMEs is to improve logistics management, implement joint logistics companies, or use third-party logistics companies to carry out logistics activities. The third-party logistics management approach is not to say that the company's logistics activities should be fully handed over to third-party companies for management, but rather that enterprises should have controlled and scientific management. That is, the company provides the origin and destination of the goods, and the specific contracting method is designed by the third-party logistics company. In the carrier process, companies can view logistics information at any time to achieve low-cost, high-efficiency management. Third-party logistics has many advantages that SMEs do not have. With these advantages, companies can better control logistics costs. By handing over logistics activities to third parties, companies can focus more on core components such as production and operations to reduce operating costs. At the same time, with the use of third-party logistics, companies can reduce staffing costs and reduce manpower and material costs in logistics activities such as equipment procurement by paying less. In general, companies actively innovate logistics management methods, will inevitably improve the scientific logistics management to a new level, thus saving a lot of manpower, material and financial costs for the company.

\section{Summary}

In summary, logistics management plays an important role in the development of SMEs and can be said to determine the cost of the enterprise. SMEs only recognize the importance of logistics management, and strictly follow the recommendations to change, strengthen logistics construction, scientific, efficient, and information-based ideas to build logistics, in order to truly improve the level of logistics management, reduce logistics costs, increase business profit.

\section{References}

[1] Danuta Janczewska. Marketing and Logistics Management as an Innovative Direction of Management in the SMEs Sector [J]. Przedsiebiorczosc i Zarzadzanie,2015,16(2).

[2] Amine Ghoumrassi, Gabriela Tigu. The impact of the logistics management in customer satisfaction[J]. Proceedings of the International Conference on Business Excellence, 2017, 11(1).

[3] Amine Ghoumrassi, Gabriela Tigu. The impact of the logistics management in customer satisfaction[J]. Proceedings of the International Conference on Business Excellence, 2018, 12(1).

[4] Yen-Chun Wu, Mark Goh, Chih-Hung Yuan, Shan-Huen Huang. Logistics management research collaboration in Asia[J]. The International Journal of Logistics Management, 2017, 28(1). 